\section{Photoperiodic Response of In Vitro Cannabis sativa Plants}

\author{
Melissa Moher \\ School of Environmental Sciences, University of Guelph, 50 Stone Road East, \\ Guelph, ON, N1G 2W1, Canada
}

\author{
Max Jones \\ Department of Plant Agriculture, University of Guelph, 50 Stone Road East, \\ Guelph, ON, N1G 2W1, Canada
}

\author{
Youbin Zheng \\ School of Environmental Sciences, University of Guelph, 50 Stone Road East, \\ Guelph, ON, N1G 2W1, Canada
}

Additional index words. Cannabis, dark period, flower initiation, photoperiod, tissue culture

\begin{abstract}
The majority of commercial Cannabis sativa L. (cannabis) cultivators use a 12.0hour uninterrupted dark period to induce flowering; however, scientific information to prove this is the optimal dark period for all genotypes is lacking. Knowing genotypespecific photoperiods may help to promote growth by providing the optimal photoperiod for photosynthesis. To determine whether the floral initiation of cannabis explants respond to varied photoperiods in vitro, explants were grown under one of six photoperiod treatments: $12.0,13.2,13.8,14.4,15.0$, and 16.0 hours per day for 4 weeks. The percentage of flowering explants was highest under 12.0- and 13.2-hour treatments. There were no treatment effects on the fresh weight, final height, and growth index. Based on the results, it is recommended that an uninterrupted dark period of at least 10.8 hours (i.e., 13.2-hour photoperiod) be used to induce flowering for the ' 802 ' genotype. In vitro flowering could provide a unique and high-throughput approach to study floral/ seed development and secondary metabolism in cannabis under highly controlled conditions. Further research should determine if this response is the same on the whole-plant level.
\end{abstract}

Cannabis sativa $\mathrm{L}$. is an herbaceous, primarily short-day plant that has been used for medicinal and recreational purposes since 2800 BC (Farquhar-Smith, 2002). After decades of prohibition, it is now becoming more accepted in today's society for its medicinal effects on pain, inflammation, and epilepsy, as well as for recreational use (Small, 2017; Zheng, 2020). As of 2018, the gross domestic product (GDP) of the medicinal and recreational Canadian cannabis industry was valued at approximately $\$ 5.7$ billion (Statistics Canada, 2020), making it one of the nation's most economically important crops. Since Canada legalized cannabis extracts, topicals, tinctures, concentrates, capsules, beverages, and edibles in 2019, the Canadian cannabis industry contribution to the GDP has increased

Received for publication 16 Sept. 2020. Accepted for publication 6 Nov. 2020.

Published online 17 December 2020.

We thank Ontario Centres of Excellence and HEXO Corp. for financial support and HEXO Corp. for providing the plant material for this experiment. We also thank Sean Kyne, Rebecca Bradley, and Josh Callaghan for their assistance with the preparation of explants and media.

Y.Z. is the corresponding author. E-mail: yzheng@ uoguelph.ca.

This is an open access article distributed under the CC BY-NC-ND license (https://creativecommons. org/licenses/by-nc-nd/4.0/). quality. Given the limited amount of scientific literature regarding the photoperiodic requirements of cannabis, the optimal dark period has not been determined for the majority of genotypes.

Cannabis can be grown under various production systems, including indoor, outdoor, greenhouse, and tissue culture environments. Tissue culture has become an important tool for genetic maintenance and propagation due to its sterility, capability of mass propagation, and preservation of genetics (Feeney and Punja, 2003; Lata et al., 2009). Most cannabis micropropagation is conducted using long-day photoperiods (16.0-18.0 $\mathrm{h}$ per day), and most plants remain in the vegetative state. However, initial observations have indicated that some genotypes flower in vitro, even under long-day conditions. This demonstrates the capacity for in vitro flower development in cannabis, which is not observed in all species. In vitro flowering and seed set have been proposed in other species as a valuable tool to reduce generation time for applications in plant breeding and to study floral/seed development in a highly controlled environment (Ochatt et al., 2000). Additional aspects specific to cannabis may include the opportunity to study the regulation of secondary metabolites, production of floral tissue for plant regeneration (Piunno et al., 2019), and the potential to rapidly identify the critical photoperiod of specific genotypes.

To date, the occurrence of in vitro flowering has only been observed sporadically, and there are no reports indicating whether the response to photoperiod is similar to that of whole plants. Flowering has been demonstrated in other short-day plants in vitro, including tobacco (Altamura et al., 1991), Plumbago indica (Nitsch and Nitsch, 1967), Cuscuta reflexa (Baldev, 1962), and Kalanchöe blossfeldiana (Dickens and van Staden, 1990). However, the fact that some short-day cannabis genotypes flower under long days suggests there are other factors involved, such as plant growth regulators (Mobini et al., 2015), day/night temperatures (Adams et al., 2009), and other environmental factors.

The overall hypothesis of this study was that cannabis plants grown in vitro will respond to photoperiod by means of flower initiation. Specific objectives included: 1) to quantify plant response to photoperiod, 2) to investigate the best flowering metric for determining the optimal photoperiod, and 3) to determine whether longer photoperiods increase growth of explants.

\section{Materials and Methods}

anidentified cannabis cultivar has a critical period between 12.0 and $14.0 \mathrm{~h}$ (Clarke, 1999). Some genotypes may benefit from longer photoperiods because it allows for more photosynthesis to occur, thereby increasing plant growth, including height (Farooqi et al., 1999), nodes, stem length (Downs and Borthwick, 1956), and dry weight (Bonner, 1940), or they may reduce the time to maturity without sacrificing yield/
Plant materials. The experiment was conducted in a walk-in tissue culture chamber at the University of Guelph, Guelph, ON, Canada. Established shoot cultures of female Cannabis sativa L. '802', a high $\Delta^{9}$ tetrahydrocannabinol genotype (15\% to $20 \%$ THC; for molecular characterization see Page et al., 2020), was used as a source of explants 
by taking shoot segments from stock plants containing at least two nodes $(\approx 1.3 \mathrm{~cm}$ in length) with the leaves trimmed to approximately half the original size. Healthy explants of a uniform size were transplanted to the middle of the prepared tissue culture vessels $(23 \times 7 \times 7 \mathrm{~cm}$, We-V box; WeVitro, Guelph, ON, Canada) and equally spaced. Then, the vessels were tightly sealed with grafting tape. There were four explants in each vessel.

Media composition. A previously optimized semi-solid tissue culture medium (Page et al., 2020) that consisted of 5.32 g/L DKW basal salts and vitamins (D2470; PhytoTech Laboratories, Shawnee, KS) (Driver and Kuniyuki, 1984), $30 \mathrm{~g} / \mathrm{L}$ sucrose, $1 \mathrm{~mL} / \mathrm{L}$ plant preservative mixture (PPM) (Plant Cell Technology, Washington, DC), and $6 \mathrm{~g} / \mathrm{L}$ of agar (A360-500; Fisher Chemical, Fair Lawn, NJ) adjusted to a $\mathrm{pH}$ of 5.7 before being autoclaved was used. Approximately $200 \mathrm{~mL}$ of the medium was poured into the bottom of each sterile We-V tissue culture vessel. The medium was autoclaved for $20 \mathrm{~min}$ at $122^{\circ} \mathrm{C}$ and $20 \mathrm{psi}$.

Growing conditions and experimental design. All explants were grown under a 16.0-h photoperiod for 2 weeks before the implementation of different photoperiod treatments. During this vegetative growth period, the vessels were placed under lightemitting diode (LED) lighting covering a 0.9$\times 0.4-\mathrm{m}$ area. The LED arrays provided a photosynthetic photon flux density (PPFD) of $19.5 \pm 1.3 \mu \mathrm{mol} \cdot \mathrm{m}^{-2} \cdot \mathrm{s}^{-1}($ mean $\pm \mathrm{SD})$ and a spectral composition for blue (B; 400-500 $\mathrm{nm})$, green $(\mathrm{G} ; 500-600 \mathrm{~nm})$, and red $(\mathrm{R}$; 600-700 nm) wavebands of B23:G18:R59 (Fig. 1). The peak wavelength and full width at half maximum (FWHM) of the peaks in the $\mathrm{B}, \mathrm{G}$, and $\mathrm{R}$ wavebands were $447 \mathrm{~nm}$ and 17 $\mathrm{nm}, 520 \mathrm{~nm}$ and $34 \mathrm{~nm}$, and $656 \mathrm{~nm}$ and 15 nm, respectively. The PPFD was obtained from 20 points over the treatment area that were measured at explant height $(\approx 2 \mathrm{~cm})$. Spectrum and $P P F D$ were measured with a radiometrically calibrated spectrometer (Flame-S-XR; Ocean Optics, Dunedin, FL) with a cosine corrector attached to a $1.9-\mathrm{m} \times 400-\mathrm{nm}$ optical fiber.

After 2 weeks, explants were transferred to one of the six photoperiod treatments $(12.0,13.2,13.8,14.4,15.0$, or $16.0 \mathrm{~h}$ per day) and grown for another 4 weeks. This was accomplished using a completely randomized experimental design that was conducted twice over time (two trials). For each trial, there were four replicates (vessels) within each treatment. Each vessel had four explants (subsamples).

The environment where the vessels were placed was maintained at $25 \pm 0.4{ }^{\circ} \mathrm{C}$ (mean \pm SD) and a relative humidity of $42 \pm 4.5 \%$. Temperature and relative humidity were measured using an external data logger (HOBO UX100-011A; Onset Computer Corporation, Bourne, MA) set to record every minute. Each vessel had its own custom LED array (Fig. 3A) that provided a $P P F D$ of $50.8 \pm 1.6 \mu \mathrm{mol} \cdot \mathrm{m}^{-2} \cdot \mathrm{s}^{-1}($ mean $\pm \mathrm{sD})$ at explant height and a spectral composition of B15:G7:R78 (Fig. 2). The peak wavelength and FWHM of the peaks in B and R wavebands were $456 \mathrm{~nm}$ and $18 \mathrm{~nm}$ and $658 \mathrm{~nm}$ and $17 \mathrm{~nm}$, respectively. Each compartment had a three-dimensional printed blackout cover that prevented light carryover from nearby treatments while still allowing for air circulation through individual compartments. Panda film (Vivosun, City of Industry, CA) encompassed all compartments as an added precaution to ensure blackout conditions whenever the LED lights were off (Fig. 3B).

Time of flowering and plant growth measurements. During the 4-week photoperiod treatment, each explant was monitored daily for flower initiation. Flower initiation was determined by pistil formation at the calyx, which is a morphological event in female cannabis plants that represents a transition from vegetative to reproductive phase. For this experiment, pistil formation was deemed a successful flowering event (Fig. 4B and C). Explant height was measured weekly using ImageJ (Version 1.52q; National Institutes of Health, Bethesda, MD) from the medium level to the highest point of the explant.

After the 4-week photoperiod treatment, vessel lids were removed to measure the growth attributes of each explant. Using the height and perpendicular width measurements $(\mathrm{N}-\mathrm{S}$ and $\mathrm{E}-\mathrm{W})$, growth index was calculated following the methods of Clark and Zheng (2020): [(height $\times$ width $_{\mathrm{N}-\mathrm{S}} \times$ width $\left._{\mathrm{E}-\mathrm{W}}\right)$. For the final heights, explants were measured with the lid removed because some had reached heights above the height of the vessel (causing lateral growth due to height restrictions). Explants were destructively harvested to obtain aboveground fresh weight. Explants were removed from the medium and aboveground fresh weight was measured with an analytical balance (Met- tler Toledo AE 100; Mettler Toledo, Columbus, $\mathrm{OH}$ ). Root weight measurements were not recorded because only some explants rooted.

Statistical analysis. Data were analyzed using a generalized linear mixed model in Statistical Analysis Software, University Edition (SAS Institute Inc., Cary, NC). The different variances were accounted for by testing for trial and treatment effects. A Tukey-Kramer's test at the $95 \%$ significance level was used to determine whether there was a difference among photoperiod treatments. When there were no statistical differences among the two trials, the data were combined. Otherwise, the data were analyzed as two separate trials (i.e., days to first flower). The residuals were checked and transformed using a lognormal distribution to satisfy the assumptions.

\section{Results}

Flower initiation. The percentage of plants that flowered was highest in the 12.0and 13.2-h photoperiod treatments, with $76 \pm$ $11 \%$ and $72 \pm 9 \%$, respectively, with no significant difference between the values. As photoperiod became longer, the percentage of plants flowered decreased, with $11 \pm$ $7 \%$ of explants with the $14.4 \mathrm{~h}$ treatment. Minimal flower events occurred with the 15.0- and 16.0-h treatments $(<3 \%)$ (Fig. 5). The 12.0- and 13.2-h treatments flowered the quickest in trial 1 (6 d), whereas flowering occurred quickest with the 13.2-h treatment in trial $2(3 \mathrm{~d})$. Floral initiation of explants reached $25 \%$ and $50 \%$ quickest under the 12.0-h photoperiod, followed by the 13.2-h photoperiod, in both trials (Table 1). Explants under photoperiods of $13.8,14.4,15.0$, and $16.0 \mathrm{~h}$ did not reach $50 \%$ flowering in both trials.

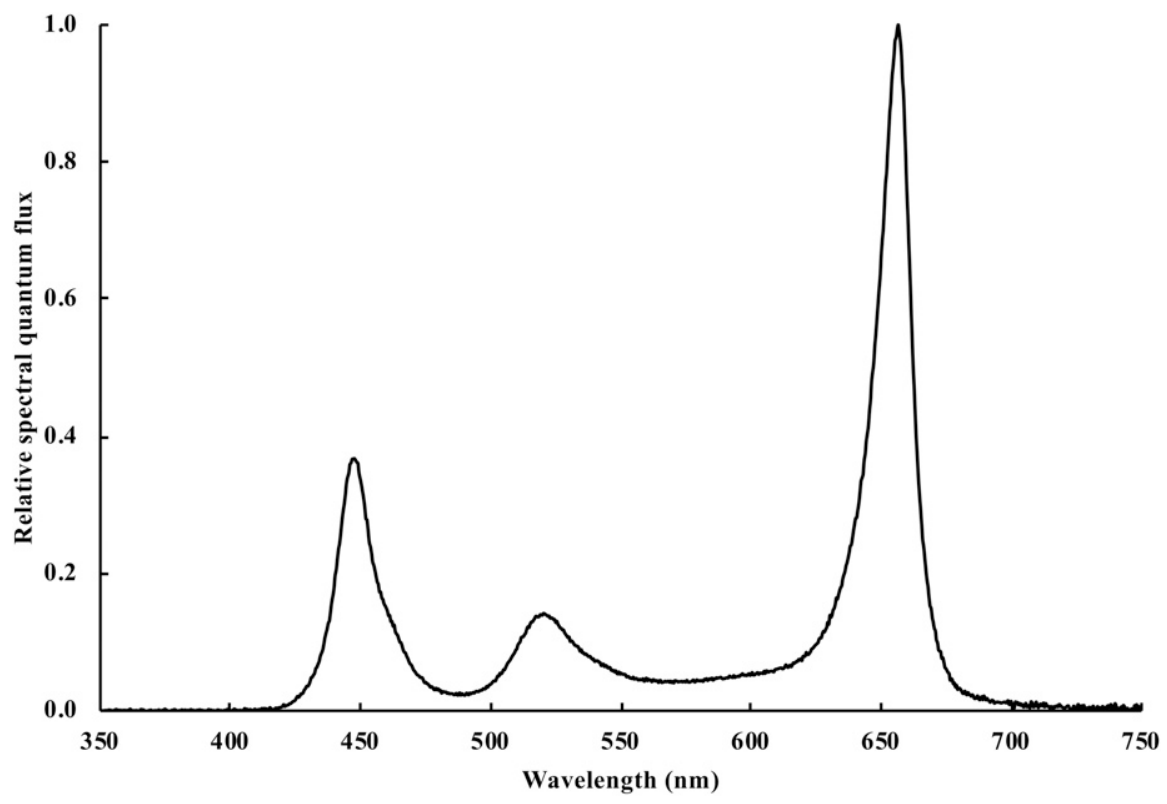

Fig. 1. Relative spectral quantum flux distribution of the light-emitting diode (LED) fixture used during the vegetative stage over the photosynthetically active radiation spectral range (i.e., $400-700 \mathrm{~nm}$ ). 
Growth. Aboveground fresh weight, final height, and growth index were not significantly affected by the different photoperiod treatments, and all had large variances. The average fresh weight was $0.4383 \pm 0.0550$ $\mathrm{g} /$ plant (range, $0.0818-1.8112 \mathrm{~g} / \mathrm{plant}$ ). The average final height was $2.9 \pm 0.16 \mathrm{~cm}$ (range, 1.7-6.0 cm). The average growth index was $0.36 \pm 0.07$ (range, 0.05-2.16).

\section{Discussion}

Flowering and photoperiod. The six photoperiod treatments were designed to have an initial $10 \%$ increase from 12.0 to $13.2 \mathrm{~h}$, and then a $5 \%$ incremental increase from $13.2 \mathrm{~h}$ onward. The 12.0- and 13.2-h photoperiods had the highest percentage (74\%) of flowered explants; this quickly decreased to only $11 \%$

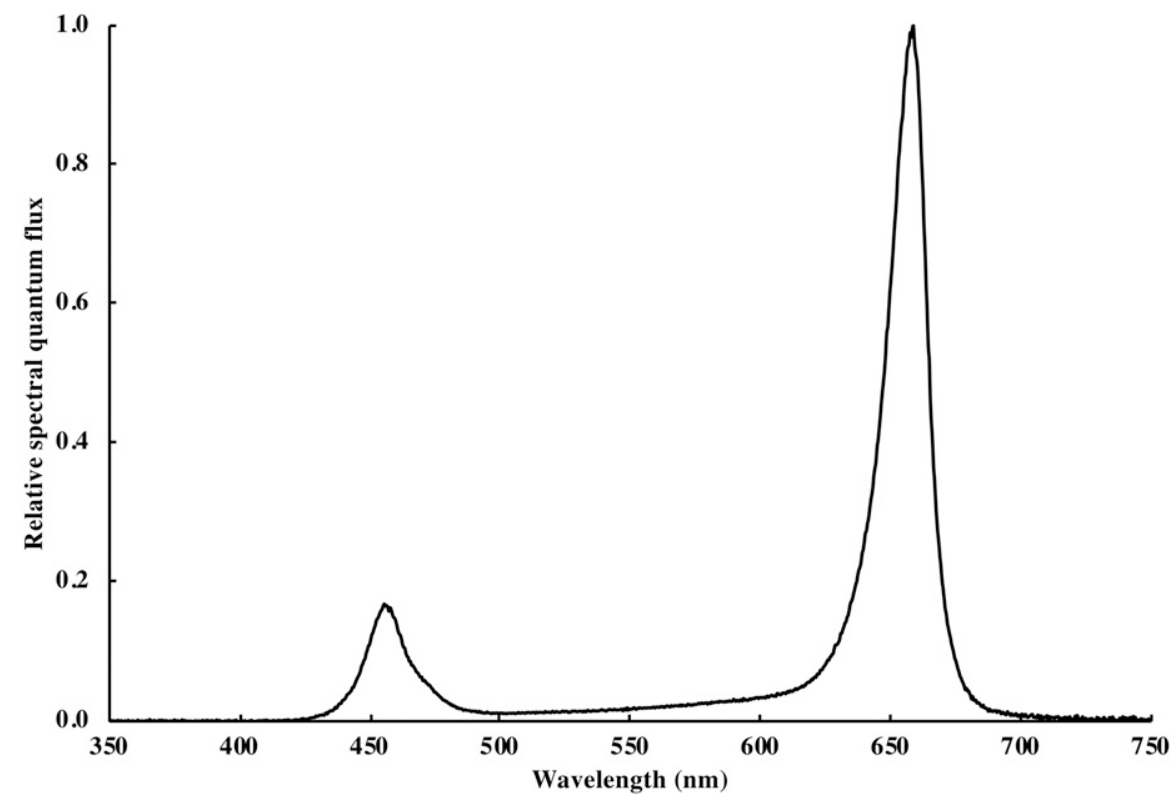

Fig. 2. Relative spectral quantum flux distribution of the custom light-emitting diode (LED) fixtures used for photoperiod treatments over the photosynthetically active radiation spectral range (i.e., 400$700 \mathrm{~nm}$ ). in the 14.4-h treatment. It eventually reached $0 \%$ in the $16-\mathrm{h}$ treatment. The percentage of flowered explants was the most reliable flowering metric and has also been used for other plant species such as strawberries (Vince-Prue and Guttridge, 1973), ornamentals (Craig and Runkle, 2013), and in vitro tomatoes (Dielen et al., 2001). Hemp studies using whole plants have reported similar results with higher percentages of flowering when grown under photoperiods less than $14.0 \mathrm{~h}$, whereas plants under 17.0 and $20.0 \mathrm{~h}$ remained vegetative (Borthwick and Scully, 1954). It was also reported that outdoorgrown hemp under longer photoperiods had delayed flowering; however, $48.3 \%$ and $47.5 \%$ still flowered under 16.0 and $19.0 \mathrm{~h}$, respectively (Borthwick and Scully, 1954). The latter study found a high percentage of

flowering under the 16.0- and 19.0-h photoperiods; however, some hemp genotypes are day-neutral and do not entirely rely on the photoperiod to induce flowering. Explants in the current study responded similarly to hemp grown in greenhouse and outdoor production, suggesting that explants may respond similarly on a whole-plant level; however, this needs further validation.

The time to first flower initiation and the average day to flower initiation were different between the two trials. The reason for the discrepancy is not known, but it could be due to different physiological conditions of the starting materials despite all attempts to maintain uniform conditions and materials. The time to first flower is not reliable because it can be misleading. For example, under the 15.0-h photoperiod treatment, the observed first explant to flower was at day 12 , but less than $5 \%$ of the explants flowered during the whole trial. Flowering under the 15.0-h photoperiod may have been a result of environmental factors rather than a photoperiod response, which is consistent with previous observations of some short-day genotypes sporadically flowering under long-day conditions in vitro. Environmental factors such as the ratio of low red to far red for cool-season grain legumes (Croser et al., 2016), higher temperatures later in the growing cycle for chrysanthemums (Carvalho et al., 2005), and exogenous growth regulators for vegetable crops (Franklin et al., 2000; Sheeja and Mandal, 2003) have reportedly enhanced floral development in terms of time and quantity. Although these factors were not present in the current study (i.e., exogenous growth regulators), there is evidence showing that flowering can occur in vitro that is independent of the photoperiod.

Of all the photoperiod treatments, only the 12.0- and 13.2-h photoperiods reached $50 \%$ flowering, which took 13 to $19 \mathrm{~d}$ during trial 1 and 19 to $22 \mathrm{~d}$ during trial 2 . The time to reach $50 \%$ flowering can be used to get an idea of the length of time required to grow the
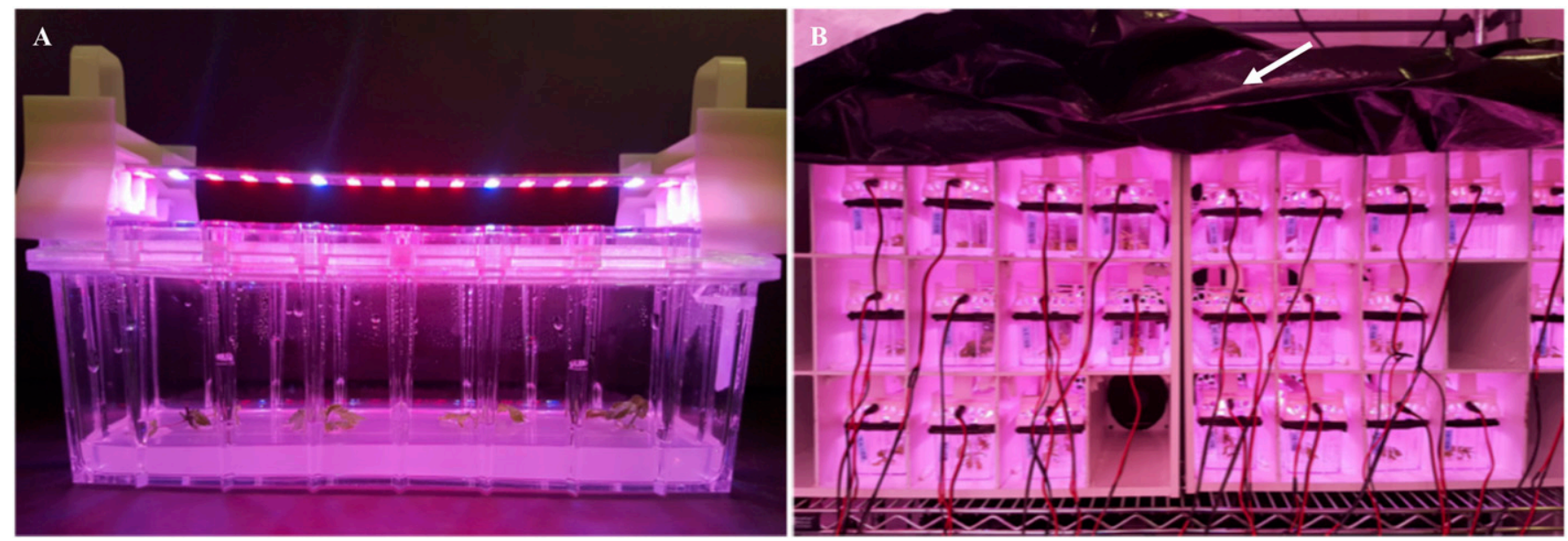

Fig. 3. (A) Tissue culture vessel with four explants with its own individual LED array. (B) Experimental setup including the vessels in individual compartments and the panda film, indicated by the white arrow, that encompasses all compartments. Each compartment had its own individual cover to prevent light spillover (not shown). 

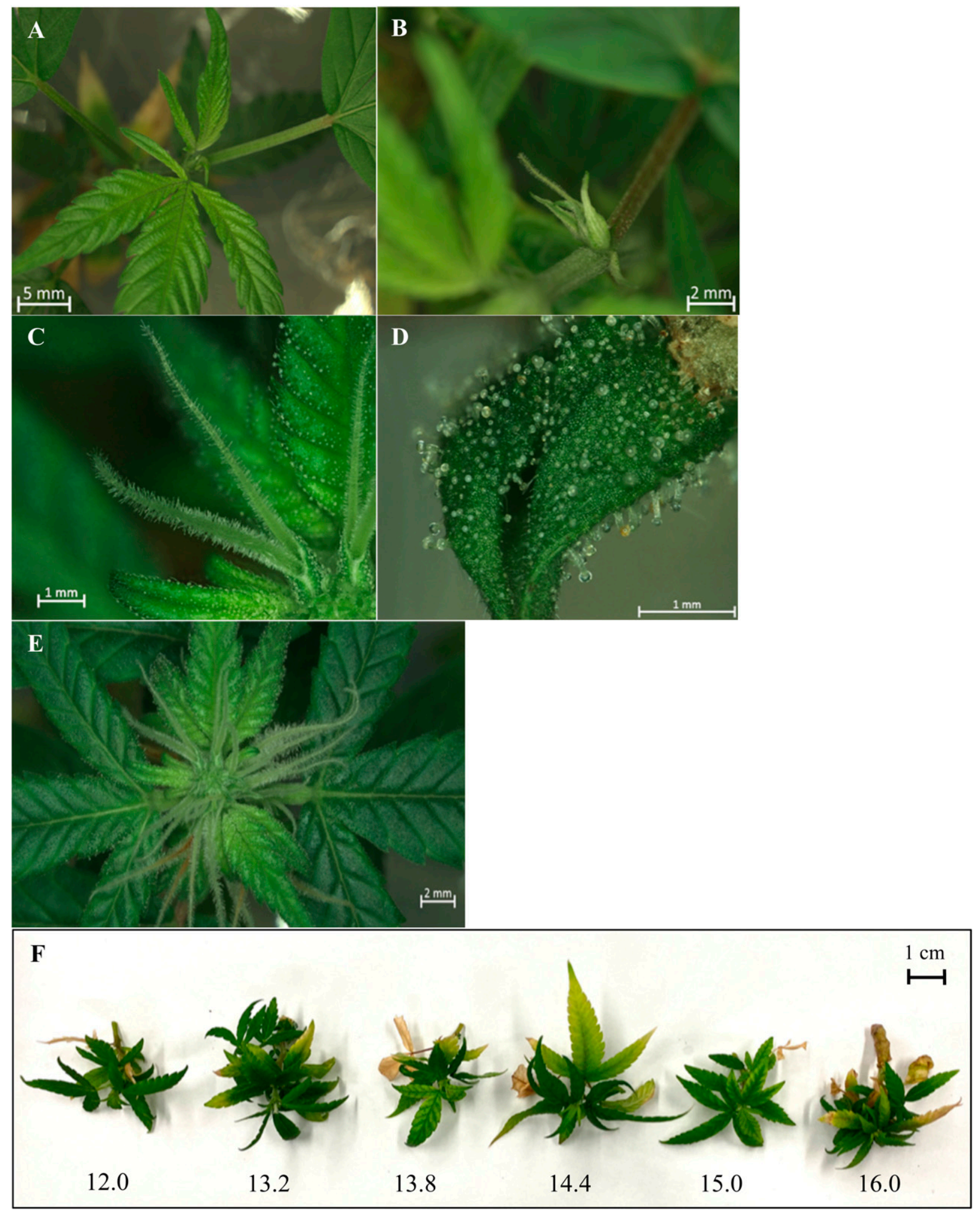

Fig. 4. Cannabis explants depicting (A) vegetative growth, (B) pistil emerging from the calyx, $(\mathbf{C})$ close-up of pistil emergence, (D) trichomes on the perigonal bract, (E) developed flower, and (F) explants at the end of the 4-week photoperiod treatments.

explants in vitro, but it should not be used to determine the most appropriate flowering metric because only two of six photoperiods reached $50 \%$ flowering. Using the number of days to reach $50 \%$ flowering can help determine the length of time to conduct in vitro experiments, which could take significantly less time than growing whole plants in a greenhouse or indoors. To put this into perspective, hemp genotypes grown in a controlled environment reached $50 \%$ floral initiation 20 to $27 \mathrm{~d}$ under a 12.0 -h photoperiod, but it took 50 to $55 \mathrm{~d}$ under a 16.0 -h photoperiod after sowing (Lisson et al., 2000). Other industrial hemp (low THC) studies showed that after sowing, plants grown under less than $13 \mathrm{~h}$ and $40 \mathrm{~min}$ took 33 to $34 \mathrm{~d}$ to exhibit the first male flower and 45 to $46 \mathrm{~d}$ to reach $50 \%$ flowering. With the same cultivar, it took $50 \mathrm{~d}$ to exhibit the first male flower and $71 \mathrm{~d}$ for $50 \%$ of plants to reach flowering when grown under a photoperiod of $14 \mathrm{~h}$ and $40 \mathrm{~min}$ (Hall et al., 2014). By using tissue culture, we can conduct an 
Table 1 . The number of days to see the first flower in any cannabis explant, average days to flower, and days for $25 \%$ and $50 \%$ of plants to flower under each photoperiod treatment for trials 1 and 2 .

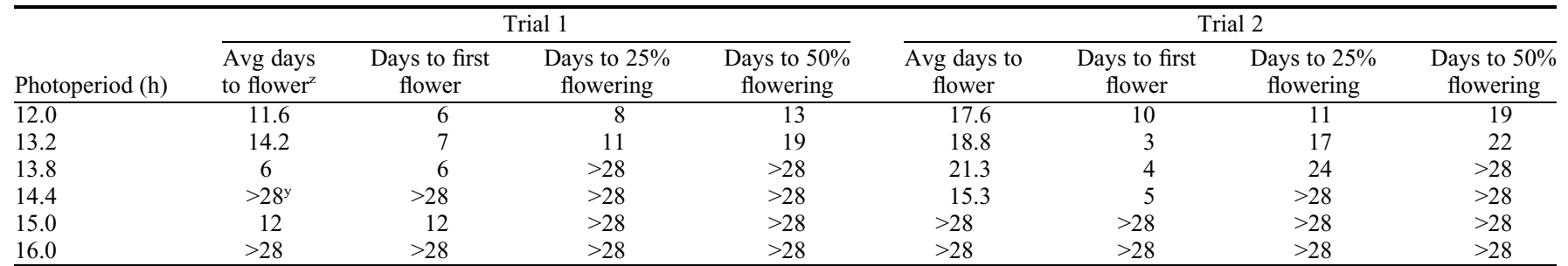

${ }^{\mathrm{z}}$ The average days to flower initiation was calculated by averaging the number of days it took for each explant to flower under each photoperiod treatment during each individual trial. This value only accounted for flowered explants.

${ }^{\mathrm{y}}$ More than $28 \mathrm{~d}(>28)$ indicates no flowering events occurred with that treatment.

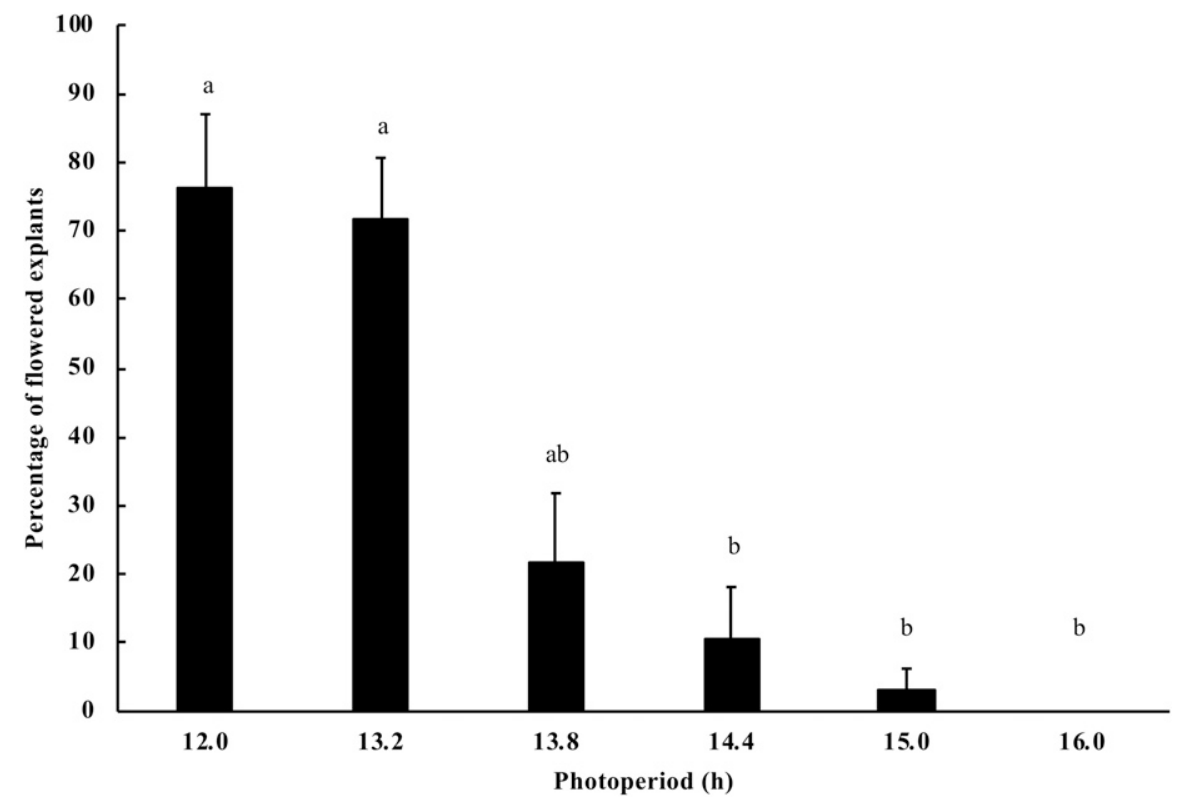

Fig. 5. Percentage (\%) of explants with flower initiated under 12.0-, 13.2-, 13.8-, 14.4-, 15.0-, and 16.0-h photoperiods. Data are means $\pm \mathrm{SE}(\mathrm{n}=8)$. Bars with different letter depict significant differences at $P \leq 0.05$ using Tukey-Kramer multiple comparisons test.

entire trial within $36 \mathrm{~d}$ (with successful treatments reaching $50 \%$ flowering) while having several photoperiods at one time to determine the genotype response to the photoperiod.

Our results showed that this cannabis genotype needs a photoperiod no longer than $13.8 \mathrm{~h}$ (i.e., 10.2-h uninterrupted dark period) per day; however, a photoperiod less than $13.2 \mathrm{~h}$ (i.e., 10.8-h uninterrupted dark period) should be used for optimal flowering based on the percentage of explants flowered. The best indicator for determining the optimal photoperiod should be based on the percentage of flowering because it provides an accurate representation of events and is not misleading to cultivators. However, whether a photoperiod less than $13.8 \mathrm{~h}$ is successful for the whole plant is unknown. Future research should use whole plants to determine the critical photoperiod needed for flower initiation for this genotype. If the results from whole plants match the results from explants, then using explants from tissue culture would be a quick and easy method for determining the critical photoperiod for different cannabis genotypes.
Growth and photoperiod. Longer photoperiods usually result in more photosynthesis and plant growth (Kozai et al., 1995; Kurilčik et al., 2008); however, to ensure floral initiation of cannabis plants, a majority of cannabis cultivators grow plants under a $12.0-\mathrm{h}$ photoperiod. For greenhouse cultivation, this means the cultivators need to block the sunlight a few hours per day during long summer days, which can be a waste of natural sunlight. For indoor cultivation, this could reduce the effectiveness of photosynthetic activity for growth purposes. The third objective of this study was to investigate whether longer photoperiods can increase plant growth while also inducing flowering. Our results showed that there was no photoperiod treatment effect on any of the measured explant growth attributes (i.e., final height, fresh weight, and growth index). Although the lack of treatment effects could be caused by the large variability among explant growth attributes and insufficient replication, it should also be highlighted that in vitro plantlets are mixotrophic and do not rely on light as their sole energy source. Due to the relatively low light intensities and supple- mental sucrose used in this study, the relative impact of extra light due to longer photoperiods is likely to be less pronounced than would be expected in a photoautotrophic system.

Another source of error that made growth rates similar among treatments was the sporadic development of roots on some explants. The presence of roots can affect the uptake of nutrients; plantlets in this study that developed roots were generally more vigorous. Since rooting only occurred on some explants and did not appear to be related to the photoperiod, this may have added a source of error that would have masked any treatment effect.

Our results show that for this cannabis genotype, the photoperiod did not significantly influence growth but did induce floral development. Further investigation using larger replication may help determine if the photoperiod influences plant growth rates in vitro. Furthermore, due to the mixotrophic nature of traditional tissue culture, this is likely to be substantially different from what would be observed in a greenhouse or growth room conditions. Further studies implementing a photoautotrophic tissue culture system may help to further elucidate these relationships; however, ultimately, whole-plant studies and a wider range of genotypes are needed.

\section{Conclusion}

The results of this study demonstrated that explants of cannabis genotype ' 802 ' can be induced to flower when the photoperiod is $13.2 \mathrm{~h}$ or less per day or, more correctly, $10.8 \mathrm{~h}$ or more per day of uninterrupted dark period. The percentage of flowering explants is the best indicator for photoperiod determination tests among the other metrics such as times for the days to first, $25 \%$, and $50 \%$ floral initiation because it provides a more accurate representation of how the explants respond under the different photoperiod treatments. The growth of explants is not a suitable method to determine the plant growth response to photoperiod because there was large variation in size and growth of explants generated from tissue culture. Future research should use whole plants to determine the critical photoperiod for flower initiation for this genotype. With further 
investigation, the use of tissue culture can be used by cultivators to save time and space to determine the specific photoperiods for their genotypes to help optimize production. Additionally, this research can help establish an in vitro system to study floral/seed development, develop in vitro breeding platforms, and investigate the regulation of secondary metabolism under highly controlled conditions.

\section{Literature Cited}

Adams, S.R., V.M. Valdés, and D. Fuller. 2009. The effects of day and night temperature on Chrysanthemum morifolium: Investigating the safe limits for temperature integration. J. Hort. Sci. Biotechnol. 84(6):604-608, doi: 10.1080/ 14620316.2009.11512573.

Altamura, M.M., G. Pasqua, B. Motiacelli, M. Tomassi, T. Archilletti, and G. Falasca. 1991. Flower formation in vitro in a quantitative short-day tobacco: Interrelation between photoperiod and infructescence development. Physiol. Plant. 82:333-338, doi: 10.1111/j.13993054.1991.tb02914.x.

Baldev, B. 1962. In vitro studies of floral induction on stem apices of Cuscuta reflexa Roxb.- a short-day plant. Ann. Bot. 26(102):173-174, doi: 10.1093/oxfordjournals.aob.a083784.

Bonner, J. 1940. Experiments on photoperiod in relation to the vegetative growth of plants. Plant Physiol. 15(2):319-325, doi: 10.1104/ pp.15.2.319.

Borthwick, H.A. and N.J. Scully. 1954. Photoperiodic responses of hemp. Bot. Gaz. 116:14-29, doi: $10.1086 / 335843$.

Carvalho, S.M.P., H. Abi-Tarabay, and E. Heuvelink. 2005. Temperature affects Chrysanthemum flower characteristics differently during three phases of the cultivation period. J. Hort. Sci. Biotechnol. 80(2):209-216, doi: 10.1080/ 14620316.2005.11511919.

Clark, M.J. and Y. Zheng. 2020. Fertilization methods for organic and conventional potted blueberry plants. HortScience 55(3):304-309, doi: 10.21273/HORTSCI14416-19.

Clarke, R.C. 1999. Botany of the genus Cannabis, p. 1-20. In: P. Ranalli (ed.). Advances in hemp research. Food Products Press, Binghamton, NY.

Cosentino, S.L., G. Testa, D. Scordia, and V. Copani. 2012. Sowing time and prediction of flowering of different hemp (Cannabis sativa $\mathrm{L}$.) genotypes in southern Europe. Ind. Crops Prod. 37(1):20-33, doi: 10.1016/j.indcrop.2011.11.017.

Craig, D.S. and E.S. Runkle. 2013. A moderate to high red to far-red light ratio from light-emitting diodes controls flowering of short-day plants. J. Amer. Soc. Hort. Sci. 138(3):167-172, doi: 10.21273/JASHS.138.3.167.

Croser, J.S., M. Pazos-Navarro, R.G. Bennett, S. Tschirren, K. Edwards, W. Erskine, R. Creasy, and F.M. Ribalta. 2016. Time to flowering of temperate pulses in vivo and generation turnover in vivo-in vitro of narrow-leaf lupin accelerated by low red to far-red ratio and high intensity in the far-red region. Plant Cell Tiss.
Org. 127:591-599, doi: 10.1007/s11240-0161092-4.

de Meijer, E.P.M. and L.C.P. Keizer. 1994. Variation of cannabis for phenological development and stem elongation in relation to stem production. Field Crops Res. 38:37-46, doi: 10.1016/ 0378-4290(94)90030-2.

Dickens, C.W.S. and J. van Staden. 1990. The in vitro flowering of Kalanchöe blossfeldiana Poellniz. II. The effects of growth regulators and gallic acid. Plant Cell Physiol. 31(6):757762, doi: 10.1093/oxfordjournals.pcp.a077975.

Dielen, V., V. Lecouvet, S. Dupont, and J.M. Kinet. 2001. In vitro control of floral transition in tomato (Lycopersicon esculentum Mill.), the model for autonomously flowering plants, using the late flowering uniflora mutant. J. Expt. Bot. 52(357):715-723, doi: 10.1093/jexbot/ 52.357 .715

Driver, J. and A. Kuniyuki. 1984. In vitro propagation of Paradox walnut rootstock. HortScience 19(4):507-509.

Downs, R.J. and H.A. Borthwick. 1956. Effects of photoperiod on growth of trees. Bot. Gaz. 117(4):310-326, doi: 10.1086/335918.

Farooqi, A.H.A., N.S. Sangwan, and R.S. Sangwan. 1999. Effect of different photoperiodic regimes on growth, flowering and essential oil in Mentha species. Plant Growth Regulat. 29:181-187, doi: 10.1023/A:1006248019007.

Farquhar-Smith, W.P. 2002. Pain and cannabinoids: Science and evidence. Pain Rev. 9:4167, doi: 10.1191/0968130202pr188ra.

Feeney, M. and Z.K. Punja. 2003. Tissue culture and agrobacterium-mediated transformation of hemp (Cannabis sativa L.). In Vitro Cell. Dev. Biol. Plant 39:578-585, doi: 10.1079/ IVP2003454.

Franklin, G., P.K. Pius, and S. Ignacimuthu. 2000 Factors affecting in vitro flowering and fruiting of green pea (Pisum sativum L.). Euphytica 115:65-74, doi: 10.1023/A:1003982900117.

Hall, J., S.P. Bhattarai, and D.J. Midmore. 2014. The effects of photoperiod on phenological development and yields of industrial hemp. J. Nat. Fibers 11:87-106, doi: 10.1080/15440478.2013.846840.

Kozai, T., K. Watanabe, and B.R. Jeong. 1995. Stem elongation and growth of Solanum tuberosum L. in vitro in response to photosynthetic photon flux, photoperiod and difference in photoperiod and dark period temperatures. Scientia Hort. 64:1-9, doi: 10.1016/03044238(95)00828-4.

Kurilčik, A., S. Dapkūnienè, G. Kurilčik, S. Žilinskaitè, A. Žukauskas, and P. Duchovskis. 2008. Effect of the photoperiod duration on the growth of Chrysanthemum plantlets in vitro. Sodinink. Darzinink. 27(2):39-46.

Lata, H., S. Chandra, I. Khan, and M.A. Elsohly. 2009. Thidiazuron-induced high-frequency direct shoot organogenesis of Cannabis sativa $\mathrm{L}$. In Vitro Cell. Dev. Biol. Plant 45(1):12-19, doi: 10.1007/s11627-008-9167-5.

Lisson, S.N., N.J. Mendham, and P.S. Carberry. 2000. Development of a hemp (Cannabis sativa $\mathrm{L}$.) simulation model 2 . The flowering response of two hemp cultivars to photoperiod.
Austral. J. Expt. Agr. 40:413-417, doi: 10.1071/EA99059.

Lumsden, P.J. and D. Vince-Prue. 1984. The perception of dusk signals in photoperiodic timemeasurement. Physiol. Plant. 60:427-432, doi: 10.1111/j.1399-3054.1984.tb06087.x.

Mobini, S.H., M. Lulsdorf, T.D. Warkentin, and A. Vandenberg. 2015. Plant growth regulators improve in vitro flowering and rapid generation advancement in lentil and faba bean. In Vitro Cell. Dev. Biol. Plant 51:71-79, doi: 10.1007/ s11627-014-9647-8.

Nitsch, C. and J.P. Nitsch. 1967. The induction of flowering in vitro in stem segments of Plumbago indica L. Planta 72:355-370, doi: 10.1007/BF00390146.

Ochatt, S.J., C. Pontécaille, and M. Rancillac. 2000. The growth regulators used for bud regeneration and shoot rooting affect the competence for flowering and seed set in regenerated plants of protein peas. In Vitro Cell. Dev. Biol. Plant 36(3):188-193, doi: 10.1007/ s11627-000-0035-1.

Page, S.R.G., A.S. Monthony, and A.M.P. Jones. 2020. Basal media optimization for the micropropagation and callogenesis of Cannabis sativa L. BioRxiv. doi: 10.1101/2020.02.07.939181.

Piunno, K.F., G. Golenia, E.A. Boudko, C. Downey, and A.M.P. Jones. 2019. Regeneration of shoots from immature and mature inflorescences of Cannabis sativa. Can. J. Plant Sci. 99:556-559, doi: 10.1139/cjps-2018-0308.

Sengloung, T., L. Kaveeta, and W. Nanakorn. 2009. Effect of sowing date on growth and development of Thai hemp (Cannabis sativa L.). Kasetsart J. (Nat. Sci.). 43:423-431.

Sheeja, T.E. and A.B. Mandal. 2003. In vitro flowering and fruiting in tomato (Lycopersicon esculentum Mill.). Asia Pac. J. Mol. Biol. Biotechnol. 11(1):37-42.

Small, E. 2017. Medical marijuana: Theory and practice, p. 275-350. Cannabis: A complete guide. CRC Press, Boca Raton, FL.

Statistics Canada. 2020. Table 36-10-0434-01 Gross domestic product (GDP) at basic prices, by industry, monthly (x 1,000,000). 25 Oct. 2020. <https://www150.statcan.gc.ca/t1/tb11/ en/tv.action?pid=3610043401\&pickMembers $\%$ $5 \mathrm{~B} 0 \% 5 \mathrm{D}=2.1$ \&pickMembers $\% 5 \mathrm{~B} 1 \% 5 \mathrm{D}=3.1 \&$ cubeTimeFrame.startMonth $=01 \&$ cubeTimeFrame. startYear $=2018 \&$ cubeTimeFrame.endMonth $=$ 07\&cubeTimeFrame.endYear $=2020 \&$ reference Periods $=20180101 \% 2$ C20200701 $>$. doi: 10.25318 / 1810000401-eng.

Struik, P.C., S. Amaducci, M.J. Bullard, N.C. Stutterheim, G. Venturi, and H.T.H. Cromack. 2000. Agronomy of fibre hemp (Cannabis sativa L.) in Europe. Ind. Crops Prod. 11:107-118, doi: $10.1016 /$ S0926-6690(99) 00048-5.

Vince-Prue, D. and C.G. Guttridge. 1973. Floral initiation in strawberry: Spectral evidence for the regulation of flowering by long-day inhibition. Planta 110:165-172, doi: 10.1007/ BF00384839.

Zheng, Y. 2020. Soilless production of drug-type Cannabis sativa. Acta Hort. (In press). 